\title{
Применение диодов Шоттки в терагерцовом частотном диапазоне
}

\author{
() Н.А. Торхов ${ }^{1,2}$, Л.И. Бабак ${ }^{2}$, А.А. Коколов ${ }^{2}$ \\ ${ }^{1}$ Научно-исследовательский институт полупроводниковых приборов АО „НИИПП“, \\ 634034 Томск, Россия \\ ${ }^{2}$ Томский университет систем управления и радиоэлектроники, \\ 634034 Томск, Россия \\ E-mail: trkf@mail.ru
}

Поступила в Редакцию 15 июля 2019 г.

В окончательной редакции 22 июля 2019 г.

Принята к публикации 22 июля 2019 г.

Более широкие возможности оптимизации конструктивных и электрофизических параметров кристаллов диодов Шоттки, выполненных по планарным технологиям „Меза-подложка“ и „Меза-Меза“ с анодными выводами в виде воздушного моста с вискером одновременно с использованием более совершенных компактных моделей, позволяют максимально эффективно использовать физические возможности контактов Шоттки при проектировании монолитных интегральных схем по диодным технологиям, повысить их надежность и преодолеть значительное отставание полупроводниковой электроники от оптоэлектроники в терагерцовом частотном диапазоне.

Ключевые слова: СВЧ, КВЧ, ТГц, компактная модель, планарный диод Шоттки, вискер, воздушный мост.

DOI: 10.21883/FTP.2019.12.48630.9215

\section{1. Введение}

Предельные рабочие частоты $f_{\mathrm{L}}$ (L - Limit) coвременных контактов металл-полупроводник (M-П) с барьером Шоттки (БШ) могут достигать инфракрасного (ИК) частотного диапазона длин электромагнитных волн - сотен терагерц (ТГц) [1,2]. Между тем значительное отставание в рабочих радиочастотах (частотных диапазонах) полупроводниковой электроники $\left(f_{\mathrm{T}}<3\right.$ ТГц) [3-5] от полупроводниковой оптоэлектроники $\left(f_{\mathrm{T}} \leq 100\right.$ ТГц $)[6]$ в значительной степени связано с влиянием приборных ограничений из-за недостаточного совершенства конструкций диодных кристаллов $[7,8]$, их компактных $[8,9]$ и физических $[10,11]$ моделей, используемых при разработке монолитных интегральных схем (МИС) по диодным технологиям.

Умение эффективно управлять электрофизическими свойствами и приборными характеристиками таких диодов в терагерцовом диапазоне открывает широкие возможности их использования в различных областях фундаментальной науки и техники: для измерения различных физико-химических и электрофизических параметров объектов, генерации, распространения и поглощения терагерцового излучения, его взаимодействия с объектами, спектральных исследованиях, контроля и управления быстропротекающими процессами, детектирования элементарных частиц и квантов излучения, взаимодействия с биологическими объектами на генном и молекулярном уровнях, сверхскоростного преобразования и передачи сигналов в высокочастотных оптоволоконных и квантовых линиях связи, ближней и космической локации, радиовидении, в энергоэффективных устройствах преобразования и передачи лучистой энергии и т.д.
Из анализа электрофизических и технических характеристик диодных кристаллов следует, что для повышения рабочих частот, выполненных по диодным технологиям МИС различного назначения, не всегда нужно стремиться к использованию предельно достижимых топологических норм, например минимизации линейных размеров (диаметров $D$ ) контактов Шоттки. Анализ компактных моделей в виде эквивалентных схем (ЭС) позволяет определить и оптимизировать влияние, оказываемое отдельными конструктивными элементами диодного кристалла (далее - диода) на его работу в конкретном частотном диапазоне. Также использование ЭС-моделей позволяет более точно учесть влияние паразитных элементов и скомпенсировать их нежелательное влияние при проектировании МИС. Такой подход позволяет с максимальной эффективностью использовать физические возможности контактов М-П с БШ в несколько раз больших диаметров и таким образом повысить надежность выполненных на их основе электронных устройств в сверхвысокочастотном (СВЧ), крайне высокочастотном (КВЧ) и терагерцовом (ТГц) частотном диапазонах электромагнитных колебаний (далее - СВЧ, КВЧ и ТГц диапазоны).

В большинстве случаев увеличение линейных размеров контактов Шоттки и сечений их выводов приводит к повышению надежности диодов Шоттки, связанной с большей устойчивостью диодных конструкций к статическому электричеству, повышением механической прочности и т.д. В качестве примера можно привести производимые по диодным технологиям работающие в терагерцовых частотных диапазонах генерирующие и преобразующие устройства фирмы „Virginia Diodes Inc." (WR1.0AMC-S, WR1.0AMC-M и WR1.0AMC-L с $f_{\mathrm{T}}<1.5$ ТГц) $[12]$. 
Необходимость в совершенствовании конструкции и ЭС обусловлена тем, что линейные размеры используемых в настоящее время полупроводниковых (например, арсенид-галлиевых GaAs, фосфид-индиевых InP и нитрид-галлиевых $\mathrm{GaN}$ ) бескорпусных кристаллов планарных детекторных, смесительных и умножительных диодов в СВЧ, КВЧ и ТГц диапазонах могут достигать сотен микрометров. С учетом относительной диэлектрической проницаемости $\varepsilon$ размер 1/12 длины электромагнитной волны $\lambda$ в диодном кристалле может быть соизмерим, или даже меньше линейных размеров некоторых его конструктивных элементов. Например, в арсениде галлия (GaAs) с $\varepsilon_{\mathrm{GaAs}} \approx 12.64 \lambda_{\mathrm{GaAs}} / 12$ составляет $\sim 23.5$ мкм для 300 ГГц и $\sim 7$ мкм для 1 ТГц, что, например, значительно меньше размеров выпускаемых промышленно планарных СВЧ диодов с расширенным контактом и балочным выводом - балочные диоды. Поэтому для адекватного описания характеристик диодов в СВЧ, КВЧ и ТГц диапазонах необходимо учесть влияние всех, даже имеющих такие малые размеры его конструктивных элементов, что обеспечивается введением в ЭС дополнительных эквивалентных элементов с учетом электромагнитных особенностей их взаимодействия.

К основным недостаткам известных компактных моделей планарных диодов диапазонов дециметровых и сантиметровых волн (например, [13,14]), ограничивающих их эффективное применение в более высокочастотных СВЧ, КВЧ и ТГц диапазонах, можно отнести недостаточное число эквивалентных элементов, входящих в состав используемых ЭС, что не позволяет описать электрические характеристики всех основных конструктивных компонентов диода в СВЧ, КВЧ и ТГц диапазонах.

Исходя из вышеизложенного в статье исследуются характеристики новых конструкций кристаллов планарных диодов Шоттки и их новая малосигнальная компактная модель способная более точно описывать работу диодов в СВЧ, КВЧ и ТГц частотном диапазоне.

Таким образом, ожидается, что использование более подробной ЭС (более совершенной компактной модели) позволит значительно повысить надежность приборов в СВЧ и КВЧ диапазонах и преодолеть частотное отставание электроники от оптоэлектроники в терагерцовом частотном диапазоне.

\section{2. Способ изготовления диодных кристаллов}

Основными недостатками конструкции балочных диодов Шоттки являются большая паразитная емкость шляпки расширенного контакта, ограничение максимального сечения $5 \times 3$ мкм балочного вывода (толщина ограничена разрастанием в стороны гальванического утолщения балки, а ее ширина - возможностями химического травления под ней разделительной канавки), определяющее во многом чувствительность диода к статическому электричеству, и конструктивно-технологические ограничения по минимальным значениям последовательных сопротивлений $R_{S}$ (расширенный контакт Шоттки нельзя близко разместить около омического контакта (ОК) во избежание сращивания их гальванических утолщений).

Для преодоления вышеперечисленных недостатков были разработаны более совершенные диоды, сочетающие в себе достоинства классических диодов Шоттки с прижимными вискерами и монолитных планарных конструкций диодных кристаллов с воздушными выводами, обеспечивающие перед балочными диодами ряд конструктивно-технологических преимуществ, которые позволяют не только исключить недостатки балочных диодов, но и обеспечить диодным конструкциям более широкие конструктивно-технологические возможности для достижения предельных технико-эксплуатационных характеристик.

В общем случае в конструкции диода различают внутренние и внешние конструктивные элементы.

К внутренним конструктивным элементам диода будем относить такие активные и пассивные элементы его конструкции, без которых он теряет свои основные функциональные свойства. Например, отсутствие барьера Шоттки или омического контакта приводит к потере диодом всех основных функциональных свойств.

К внешним конструктивным элементам будем относить все остальные пассивные реактивные и диссипативные конструктивные элементы диода: воздушные и балочные выводы, вискеры, контактные площадки, межэлементную изоляцию, подложку и другие. Создаваемые внешними элементами электрические характеристики обычно относят к паразитным электрическим характеристикам диода, ухудшающим его высокочастотные свойства.

Основные технологические этапы изготовления кристаллов планарных диодов Шоттки (далее - диодов) по конструкциям „Меза-подложка“ и „Меза-Меза“ с выводами в виде воздушных мостов (воздушный вывод) с вискерами представлены на рис. 1.

Для изготовления диодов использовались эпитаксиальные структуры, представляющие собой полуизолирующую подложку арсенида галлия $i$-GaAs $\{001\}$ I (рис. 1) с выращенными на ней методом МОС-гидридной эпитаксии контактным $n^{+}-\mathrm{GaAs}$ слоем 2 толщиной 5 мкм и барьерным слоем $n$-GaAs 3 толщиной 80 нм. Уровень легирования слоя $n^{+}$-GaAs составил $2 \cdot 10^{18} \mathrm{~cm}^{-3}$, а слоя $n$-GaAs $-7 \cdot 10^{16} \mathrm{~cm}^{-3}$.

По конструкции „Меза-подложка“ технологический маршрут изготовления диодов Шоттки включал в себя следующий набор основных технологических операций (рис. 1, I): нанесение на поверхность барьерного слоя $n$-GaAs 3 диэлектрической пленки $\mathrm{SiO}_{2} 4$ толщиной $d\left(\mathrm{SiO}_{2}\right)=0.2$ мкм, в которой по маске фоторезиста 5 химическим травлением $\mathrm{SiO}_{2}$ и слоя $n$-GaAs вскрывалось окно $U$-образной формы 6 , в котором методом 

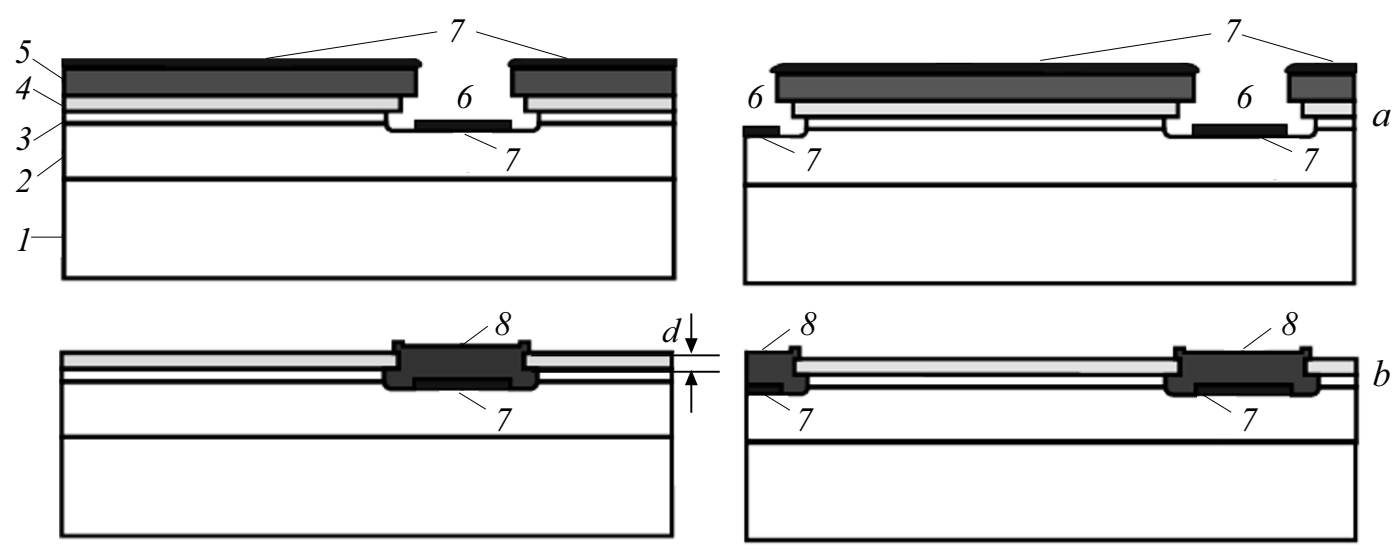

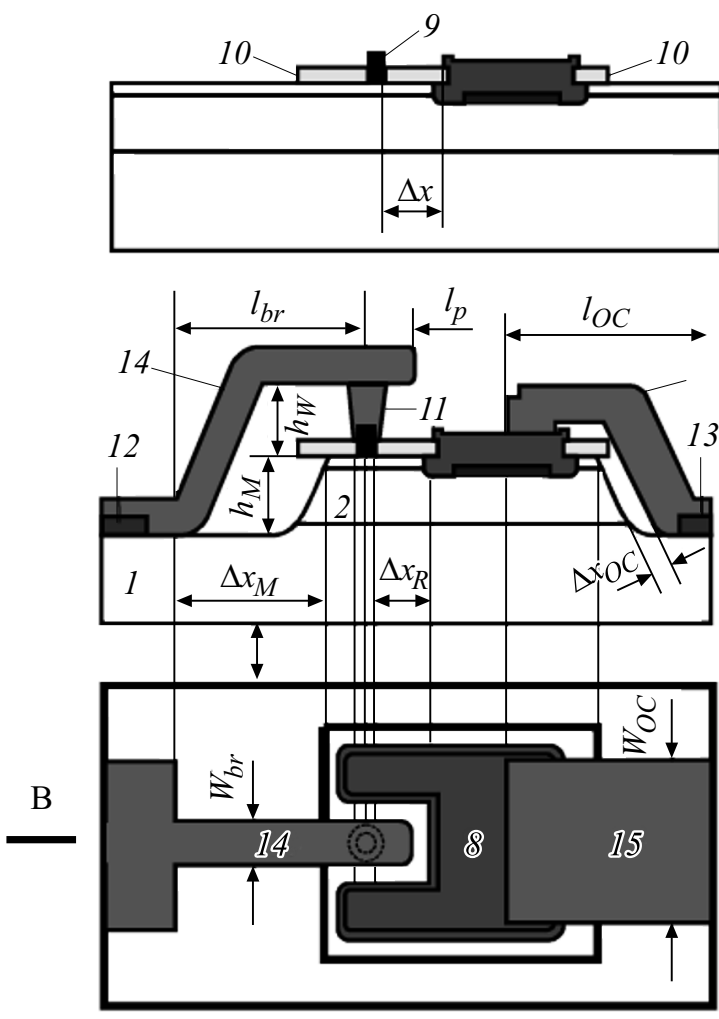

I
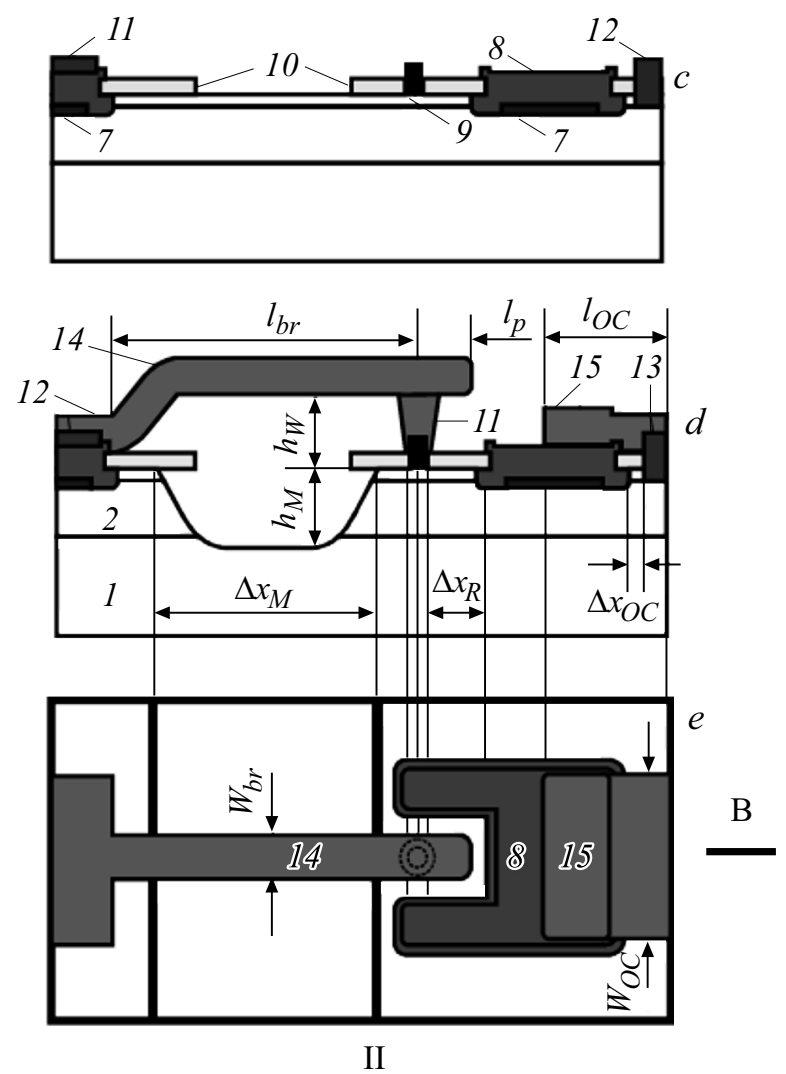

Рис. 1. Основные ключевые моменты двух вариантов изготовления планарных диодов с анодным выводом в виде воздушного моста с вискером по технологии „Меза-подложка“ (I) и „Меза-Меза“ (II).

взрывной литографии формировался сплавной омический контакт AuGeNi 7 с последующим гальваническим утолщением золотом $8-$ катод, формирование в окне $\mathrm{SiO}_{2}$ на расстоянии $\Delta x_{R}$ от катода к барьерному $n$-слою 3 анодного контакта Шоттки диаметром $D=0.8$ мкм на основе металлизации RhPt 50+50 нм 9 с последующим гальваническим утолщением золотом $\mathrm{Au}$ до $d(\mathrm{Au})=300 \mathrm{Hм}$, формирование диэлектрической маски 10, формирование вискера 11 высотой $h_{W}=3$ мкм, после чего по маске диэлектрической пленки травилась меза глубиной $h_{M}=6-7$ мкм и на полуизолирующем слое $i$-GaAs на расстоянии $\Delta x_{M}$ от основания мезы формировалась анодная 12, а с другой стороны мезы на расстоянии $\Delta x_{O C}$ катодная 13 контактные площадки.

После этого анодную контактную площадку 12 соединяли золотым воздушным мостом 14 толщиной 3 мкм, длиной $l_{b r}=24$ мкм и шириной $W_{b r}=12$ мкм с вискером 11, а катод - золотым воздушным мостом 15 толщиной 3 мкм, длиной $l_{O C}=26$ мкм и шириной $W_{O C}=36$ мкм с катодной контактной площадкой 13.

По конструкции „Меза-Меза“ технологический маршрут изготовления диодов Шоттки с небольшими поправками состоял из аналогичного набора технологических операций (рис. 1, II). 


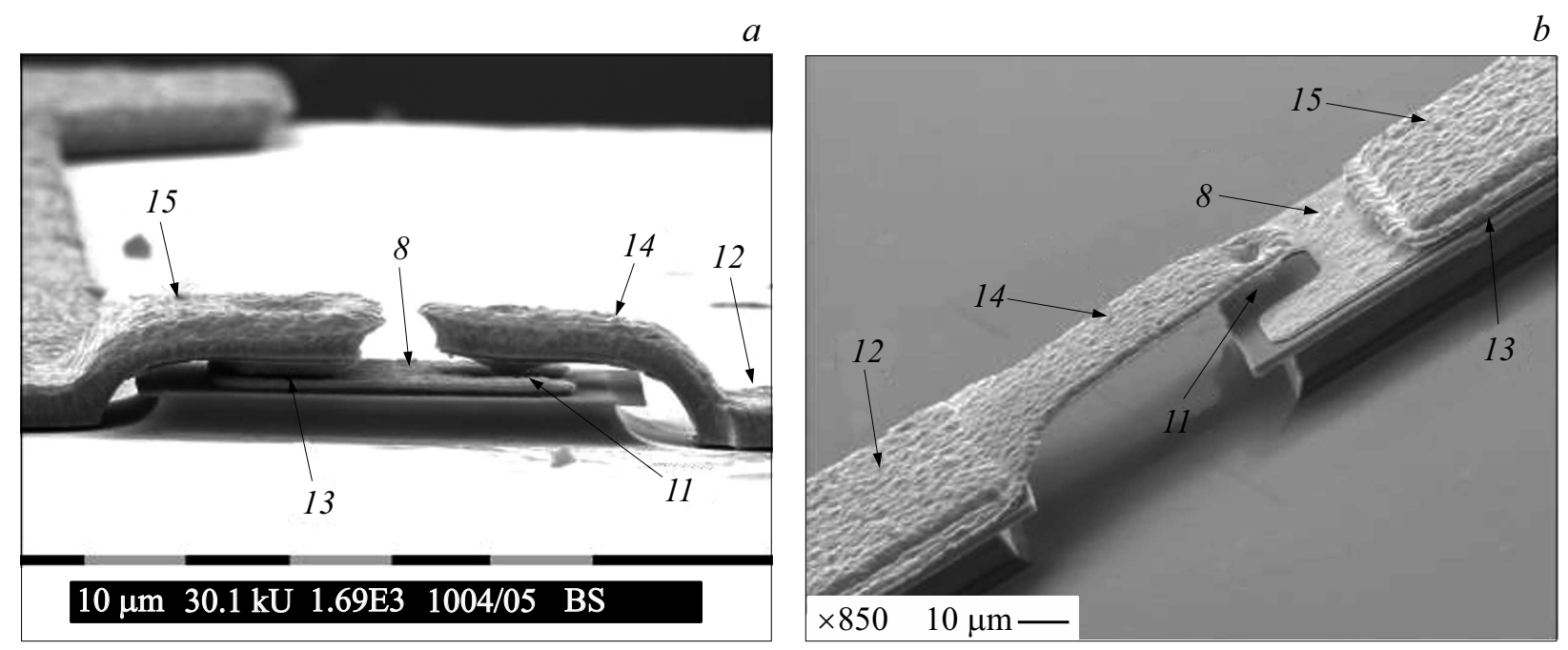

Рис. 2. Электронно-микроскопические изображения кристаллов планарных диодов Шоттки, выполненных по технологиям „Меза-подложка“ $(a)$ и „Меза-Меза“ $(b)$. На обоих изображениях: 8 - омический контакт, $11-$ вискер, $12-$ анодная контактная площадка, 13 - катодная контактная площадка, $14-$ анодный воздушный вывод (мост), $15-$ катодный воздушный вывод (мост).

В результате были получены планарные кристаллы диодов Шоттки с анодным воздушным выводом с вискером по конструкции „Меза-подложка“ (рис. 2,a) и „Меза-Меза“ (рис. 2, b).

Более широкие возможности у диодных конструкций „Меза-подложка“ и „Меза-Меза“ реализуются за счет возможного варьирования в более широких пределах, ответственных за высокочастотные характеристики диода таких конструктивных размеров, как $\Delta x_{R}, \Delta x_{O C}, h_{M}, h_{W}$, $l_{p}, l_{b r}, l_{O C}, W_{b r}$, и $W_{O C}[15]$.

\section{3. Компактные модели диодных кристаллов}

Известные компактные модели диодов Шоттки в виде малосигнальных ЭС (далее - просто ЭС), имеющие ограниченный набор из трех эквивалентных элементов $\left(C_{j}, I(U), R_{S}\right)$, пригодны для восстановления (экстракции) параметров эквивалентных элементов только в относительно низкочастотных диапазонах $\left(f_{\mathrm{T}} \sim 3-6\right.$ ГГц $)[16]$. Увеличение количества эквивалентных элементов до четырех за счет добавления емкости $C_{p}$ приводит к повышению частотного диапазона ЭС до $f_{\mathrm{T}}<40$ ГГц $[17]$, а добавление пятого элемента $C_{f p}$ повышает частотный диапазон ЭС уже до $f_{\mathrm{T}}<110$ ГГц [18]. Добавление индуктивности $L_{f}$ и увеличение эквивалентных элементов до шести позволяет с учетом емкостных и индуктивных паразитных параметров контактных площадок $C_{\text {pad1 }}, C_{\text {pad2 }}$ и $L_{\text {pad1 }}, L_{\text {pad2 }}$ значительно расширить частотный диапазон ЭС до $f_{\mathrm{T}}<600$ ГГц [18]. В общей сложности ЭС с шестью элементами с учетом паразитных параметров контактных площадок позволяет проектировать диодные

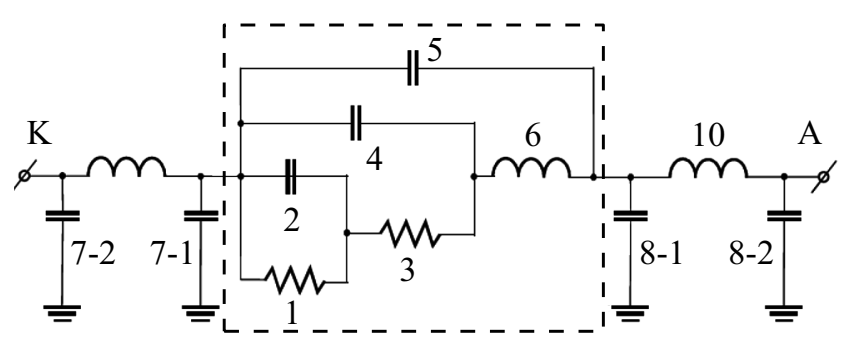

Рис. 3. ЭС известной компактной модели планарного диода Шоттки (обведено пунктиром) с эквивалентными элементами по питанию [18].

МИС и устройства на их основе уже до терагерцового диапазона частот электромагнитных колебаний $\left(f_{\mathrm{T}} \leq 2.7\right.$ ТГц $)[3-5]$. Такая ЭС содержит два нелинейных элемента: 1 - сопротивление $R_{J}$ контакта М-П с БШ, 2 - нелинейная емкость $C_{J}$, и четыре линейных элемента: 3 - последовательное сопротивление $R_{S}, 4$ емкость между анодным контактом и поверхностью $C_{f p}$, 5 - емкость анод-катод $C_{p p}, 6-$ индуктивность диода $L_{f}$, а также паразитные элементы контактных площадок: 7-1 $-C_{\mathrm{pad} 1} / 2,7-2-C_{\mathrm{pad} 1} / 2,8-1-C_{\mathrm{pad} 2} / 2$, $8-2-C_{\mathrm{pad} 2} / 2,9-L_{\mathrm{pad} 1}$ и $10-L_{\mathrm{pad} 2}($ рис. 3$)$.

Таким образом, можно выявить некоторую эмпирическую функциональную зависимость приборного ограничения частотного предела (далее просто - частотный предел $f_{\text {T) }}$ работы малосигнальной ЭС от количества $N$ образующих ее эквивалентных элементов. Исходя из литературных данных зависимость частотного предела применимости эквивалентной схемы диода $f_{\mathrm{T}}$ от количества $N$ эквивалентных элементов по аналогии с законом Мура можно ориентировочно аппроксимировать 
эмпирической зависимостью

$$
f_{\mathrm{T}}(N) \approx 43.63 \cdot \exp (N / 2.7),
$$

позволяющей прогнозировать частотные возможности малосигнальной ЭС в зависимости от числа $N$ образующих ее эквивалентных элементов.

По аналогии с классификацией конструктивных элементов диода будем классифицировать эквивалентные элементы ЭС также на внутренние и внешние описывающие электрические характеристики соответствующих внутренних и внешних его конструктивных элементов.

К внутренним эквивалентным элементам ЭС будем относить такие активные и пассивные эквивалентные элементы (далее - элементы), без которых ЭС теряет свои основные функциональные свойства. Например, при отсутствии переменного сопротивления перехода $R_{j}$ и переменной емкости $C_{j}$ ЭС диода теряет свои основные свойства.

К внешним элементам ЭС диода будем относить все остальные эквивалентные элементы, описывающие пассивные реактивные и диссипативные характеристики соответствующих его конструктивных элементов. При отсутствии даже нескольких из внешних элементов ЭС сохраняет свои основные свойства. Внешние эквивалентные элементы обычно описывают паразитные электрические характеристики диода.

Изучение конструкции диодного кристалла показало, что некоторые элементы известной ЭС (рис. 3) описывают интегральное влияние сразу нескольких внутренних и (или) внешних конструктивных элементов диода, выполненных как по технологии „Меза-подложка“, так и по технологии „Меза-Меза“. Это делает невозможным учитывать при проектировании МИС электромагнитное влияние, оказываемое на работу электрической схемы МИС каждым конструктивным элементом диода в отдельности, что значительно ухудшает точность проектирования и, как следствие, существенно снижает максимальные значения $f_{\mathrm{T}}$ проектируемых МИС. Поэтому для уточнения компактной модели диода необходимо сначала выделить более мелкие функциональные элементы его конструкции и затем описать их соответствующими дополнительными эквивалентными элементами.

Так, представленный на рис. 3 эквивалентный элемент 3 известной ЭС [18] описывает результирующее резистивное сопротивление $R_{S}$, которое образовано резистивным сопротивлением $R_{B M}$ барьерной металлизации $3-1$, сопротивлением $R_{L}$ эпитаксиальных слоев структуры $3-2$, сопротивлениями катода $R_{C} 3-3$ и вискера $R_{W} 3-4$, сопротивлением $R_{b r}$ воздушного моста 3-5, сопротивлением $R_{c t d}$ вывода катода 3-6, сопротивлением $R_{S C}$ подложки $3-7$, сопротивлениями металлизаций контактных площадок $R_{C S} 3-8$ и $R_{A S} 3-9$.

Эквивалентный элемент 4 описывает индуктивность диода $L_{f}$, образованную последовательно включенными индуктивностями воздушного моста $L_{b r} 4-1$ и вывода катода $L_{c t d} 4-2$.
Эквивалентный элемент 5 описывает интегральную электрическую емкость $C_{p p}$ диодного кристалла, образованную емкостью $C_{p p}^{\text {air }}$ между анодом и катодом по воздуху $5-1$, емкостью $C_{c t d}$ между выводом омического контакта и гетероструктурой $5-2$ и емкостью $C_{\text {sub }}$ подложки 5-3.

Эквивалентный элемент 6 описывает интегральную электрическую емкость $C_{f p}$ между анодным контактом и поверхностью, которая включает емкость $C_{i}$ между периферией анодного контакта и окружающей его поверхностью 6-1, емкость $C_{W}$ вискер-поверхность 6-1, емкость $C_{b O C}$ между шляпкой вискера и металлизацией катода 6-3 и емкость $C_{b r}$ между воздушным мостом и активными слоями мезаструктуры 6-4.

В результате совершенствование ЭС компактной модели диода в настоящей работе достигалось путем замены и добавления в известную ЭС недостающих эквивалентных элементов, описывающих пассивные реактивные и диссипативные электрические характеристики всех внутренних и внешних конструктивных элементов диода, построением электрических соединений между новыми недостающими и уже существующими эквивалентными элементами [19]. Согласно рис. 4, предложенная усовершенствованная ЭС пригодна для моделирования высокочастотных характеристик как диодов, выполненных по конструкции „Меза-подложка“, так и диодов, выполненных по конструкции „Меза-Меза“.

С учетом детального анализа конструктивные элементы внутреннего диода будем описывать шестью активными эквивалентными элементами: 1 - нелинейным сопротивлением $R_{j}, 2$ - нелинейной емкостью $C_{J}(U)$ выпрямляющего контакта, 3-1 - сопротивлением барьерной металлизации $R_{B M}, 6-1-$ электрической емкостью $C_{i}$ между периферией анодного контакта и окружающей его поверхностью, $3-2-$ сопротивлением гетероэпитаксиальных слоев $R_{L}$ между выпрямляющим и омическим контактами, $3-3-$ сопротивлением катода $R_{C}$.

Конструктивные элементы внешнего диода будем описывать шестнадцатью эквивалентными элементами: 3-4 - сопротивлением вискера $R_{W}, 3-5$ - сопротивлением воздушного моста $R_{b r}, 3-6$ - сопротивлением $R_{c t d}$ вывода катода, 3-7 - электрическим сопротивлением $R_{S C}$ подложки, $3-8-$ электрическим сопротивлением $R_{C S}$ катодной контактной площадки, $3-9$ - электрическим сопротивлением $R_{A S}$ анодной контактной площадки, $4-1$ - индуктивностью воздушного моста $L_{b r}, 4-2$ - индуктивностью $L_{c t d}$ вывода катода, 5-1 емкостью $C_{p p}^{\text {air }}$ между анодом и катодом по воздуху, 5-2 - емкостью $C_{c t d}$ между выводом омического контакта и гетероструктурой, 5-3 - емкостью подложки $C_{s u b}, 6-2-$ электрической емкостью вискерповерхность $C_{W}, 6-3-$ емкостью $C_{b O C}$ между шляпкой вискера и металлизацией катода, $6-4-$ емкостью $C_{b r}$ между воздушным мостом и гетероэпитаксиальными слоями мезаструктуры, 7 - электрической емкостью 

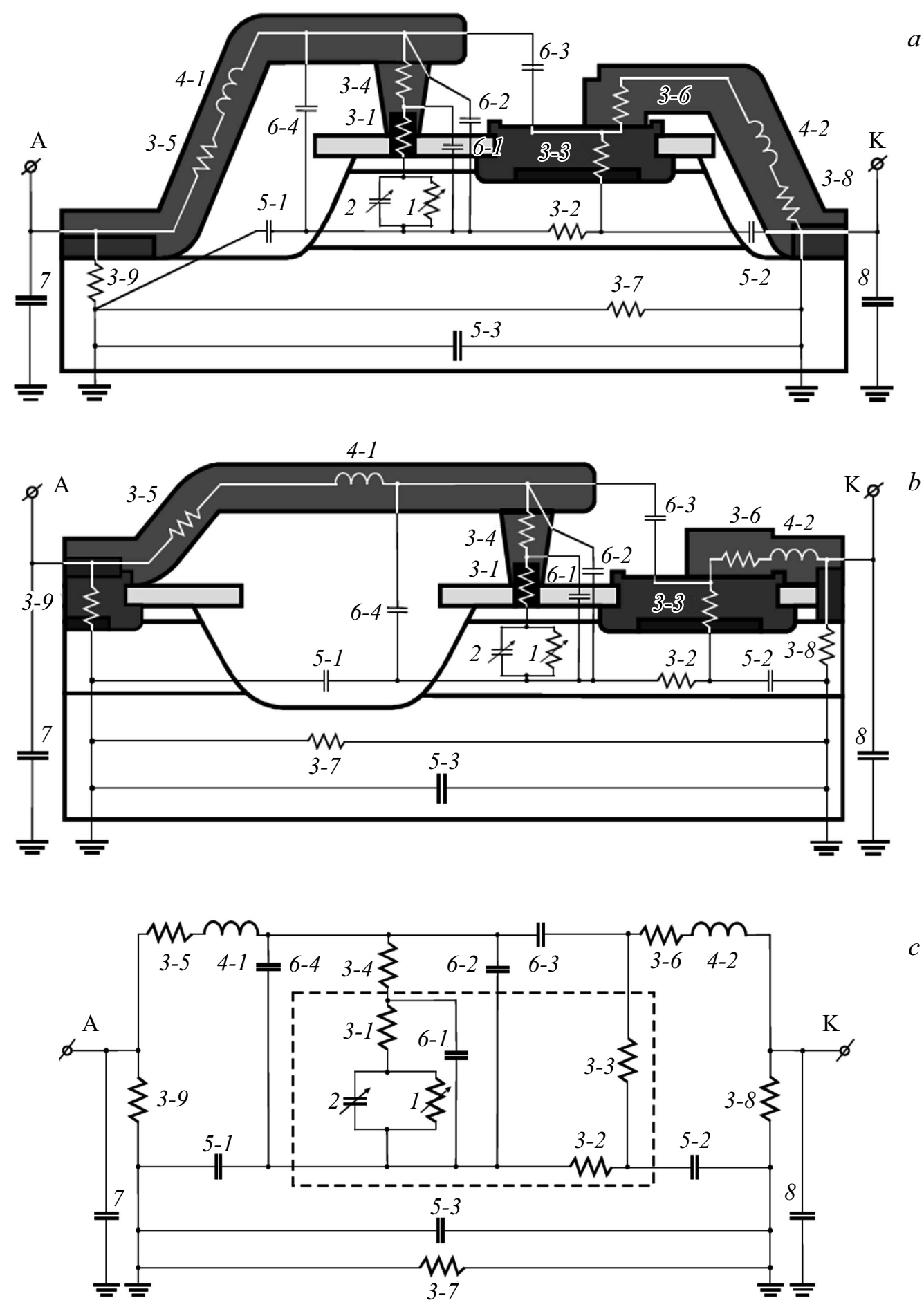

Рис. 4. Схематические изображения конструкций планарных диодов Шоттки изготовленных по технологиям „Меза-подложка“ $(a)$ и „Меза-Меза“ $(b)$ с их ЭС $(c)$. Пунктиром обведены элементы внутреннего диода.

фильтра по питанию анода $C_{\mathrm{pad} 1}$ и 8 - электрической емкостью фильтра по питанию катода $C_{\text {pad2 }}$.

Понятно, что влияние указанных факторов необходимо учитывать при проектировании по диодным технологиям МИС в виду того, что в СВЧ, КВЧ и ТГц диапазонах конструктивные элементы диода могут оказывать заметное влияние не только друг на друга, но и эффективно взаимодействовать с окружающими их конструктивными элементами МИС.

В малосигнальной ЭС балочных диодов с расширенным контактом можно выделить аналогичные эквивалентные элементы, что дает возможность использовать предложенную ЭС также и для моделирования их СВЧ, КВЧ и ТГц характеристик. 

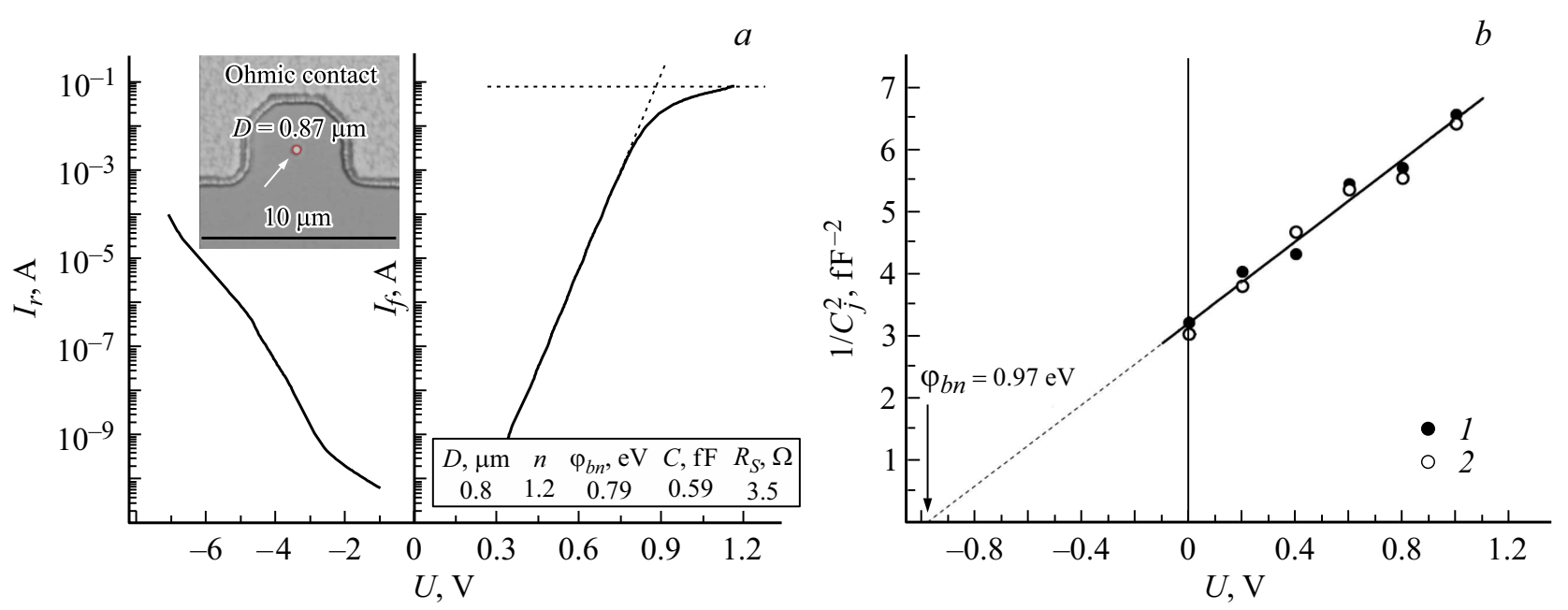

Рис. 5. Прямая $I_{f}\left(U_{f}\right)$ обратная $I_{r}\left(U_{r}\right)$ ветви статической $\mathrm{BAX} \mathrm{RhPt} / n$-GaAs контакта Шоттки $(a)$. На вставке - оптическое (ультрафиолетовое) изображение контактного окна в пленке $\mathrm{SiO}_{2}$. Зависимости $1 / C^{2} \mathrm{B \Phi X}$ от смещения $U: 1-$ экстракция, $2-$ эксперимент.

\section{4. Методика и результаты измерений}

Измерение в четырехзондовом режиме метода Кельвина статических вольт-амперных (ВАХ) и вольт-фарадных (ВФХ) характеристик барьерных контактов диаметром $D=0.87$ мкм осуществлялось на оснащенной антивибрационной и экранирующей системами зондовой станции М-150 производства Cascade Microtech при постоянной температуре $25^{\circ} \mathrm{C}$ с использованием четырех микроманипуляторов и измерителя электрических характеристик полупроводниковых приборов Agilent B-1500. Точность позиционирования используемых микроманипуляторов, оснащенных вольфрамовыми измерительными зондами с диаметром острия иглы 0.6 мкм, обеспечивала необходимый электрический контакт к субмикронным контактам Шоттки.

Несмотря на малые размеры $(D=0.87$ мкм $)$ контакта Шоттки, прямая ветвь его ВАХ имеет достаточно протяженный линейный участок $\ln \left(I\left(U_{f}\right)\right)$ в интервале токов $10^{-9}<I_{f}<10^{-4} \mathrm{~A}$ и характеризуется рекордно малыми по сравнению с [13] (контакты Шоттки Pt $/ n$-GaAs: для $D=1$ мкм, $R_{S}=15 \mathrm{Oм}, \quad C_{j}\left(N_{D}=2 \cdot 10^{17} \mathrm{~cm}^{-3}\right)$ $=1.2 \phi \Phi, n=1.18-1.23$, для $D=0.8$ мкм, $R_{S}=20$ Ом, $\left.C_{j}\left(N_{D}=2 \cdot 10^{17} \mathrm{~cm}^{-3}\right)=1.0 \phi \Phi[13]\right)$ своими параметрами: $R_{S}=3.5$ Ом, показателем идеальности $n=1.2$ при емкости перехода $C_{j}(U r=0)=0.59 \phi \Phi$ (рис. 5, $a$ ). Определенное методом ВАХ эффективное (измеряемое) значение высоты барьера $\varphi_{b m}(\mathrm{BAX})=0.79$ эВ ( $m$ - measurement), определяющее действительные параметры BAX RhPt/n-GaAs контакта, заметно меньше истинного, определенного по методу ВФХ значения $\varphi_{b n}(\mathrm{~B} \Phi \mathrm{X})=0.97$ эВ, которое превышает аналогичное значение, предсказываемое теорией термоэлектронной эмиссии $\varphi_{b n}=n \varphi_{b m}(\mathrm{BAX})=0.95$ эВ. Такое расхождение может быть вызвано значительным влиянием электростатических полей периферии контакта $[10]$, а также влиянием потенциала барьера Шоттки на прохождение над ним баллистических электронов [11].

Полученные параметры ВАХ и ВФХ позволяют оценить влияние приборного ограничения на рабочую частоту такого контакта, как $f_{\mathrm{T}} \approx 77 \mathrm{TГц} \mathrm{(2):}$

$$
f_{\mathrm{T}}=\frac{1}{2 \pi R C} .
$$

В общем случае для оценки физического частотного предела работы $f_{\mathrm{L}}$ диодов Шоттки необходимо использовать физическую модель баллистического переноса носителей электрических зарядов в современных контактах Шоттки с тонкой базой [19] с использованием новой, например [20], квантово-механической концепции одномерного законченного рассеяния, объединяющей в себе два независимых когерентно протекающих подпроцесса прохождения и отражения.

Напомним, что в квантово-механическом приближении определяемая вигнеровским (групповым) временем переноса „Phase time“ [21,22], или временем пребывания „sojourn and dwell time“" [23] скорость переноса баллистических электронов $v_{\text {ball }}$ через область потенциального барьера в нанометровых базах может значительно (например, $v_{\text {ball }}>5 \cdot 10^{7} \mathrm{~cm}^{-1}$ [24], или $v_{\text {ball }}>10^{8} \mathrm{~cm}^{-1}$ [19]) превышать их дрейфовую (с учетом процессов рассеяния) групповую скорость $v_{g}$ $\left(v_{g}<2 \cdot 10^{7} \mathrm{~cm}^{-1}[24]\right)$, определенную в рамках классической диффузионной теории. Приводимые оценки скоростей туннелирования баллистических частиц через широкие потенциальные барьеры могут существенно превышать $v_{g}[25,26]$.

Измерения малосигнальных $S$-параметров диодных кристаллов в частотном диапазоне $0.1-50$ ГГц выполнялись с использованием векторного анализатора цепей PNA-X N5245A производства Keysight Technologies на полуавтоматической зондовой станции Summit 12000 


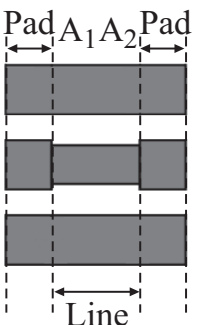

$b$

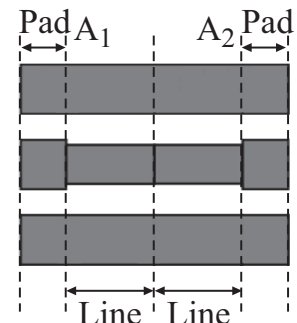

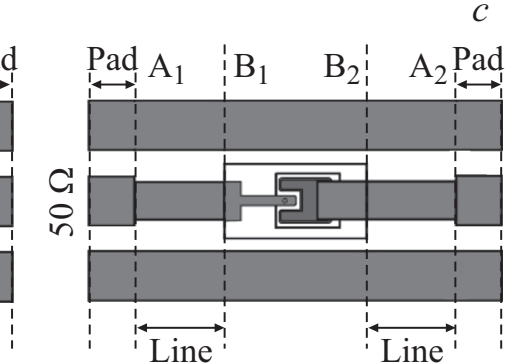

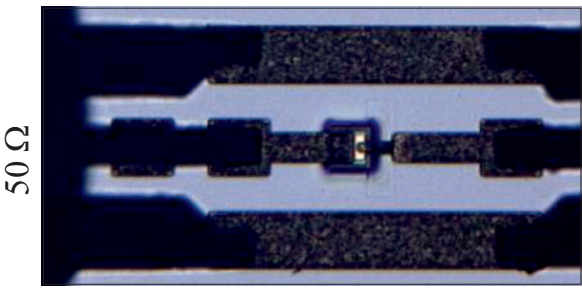

Pис. 6. Схематическое изображение тестовых элементов для проведения процедуры дэ-эмбеддинга: L-Tect $(a)$, 2LLL-Tecт $(b)$ и $\mathrm{L}-\mathrm{B}_{1} \mathrm{~B}_{2}$-L-Тест $(c)$, оптическое изображение чипа $\mathrm{L}-\mathrm{B}_{1} \mathrm{~B}_{2}$-L-Теста с измерительными СВЧ-головками $(d)$.

$a$
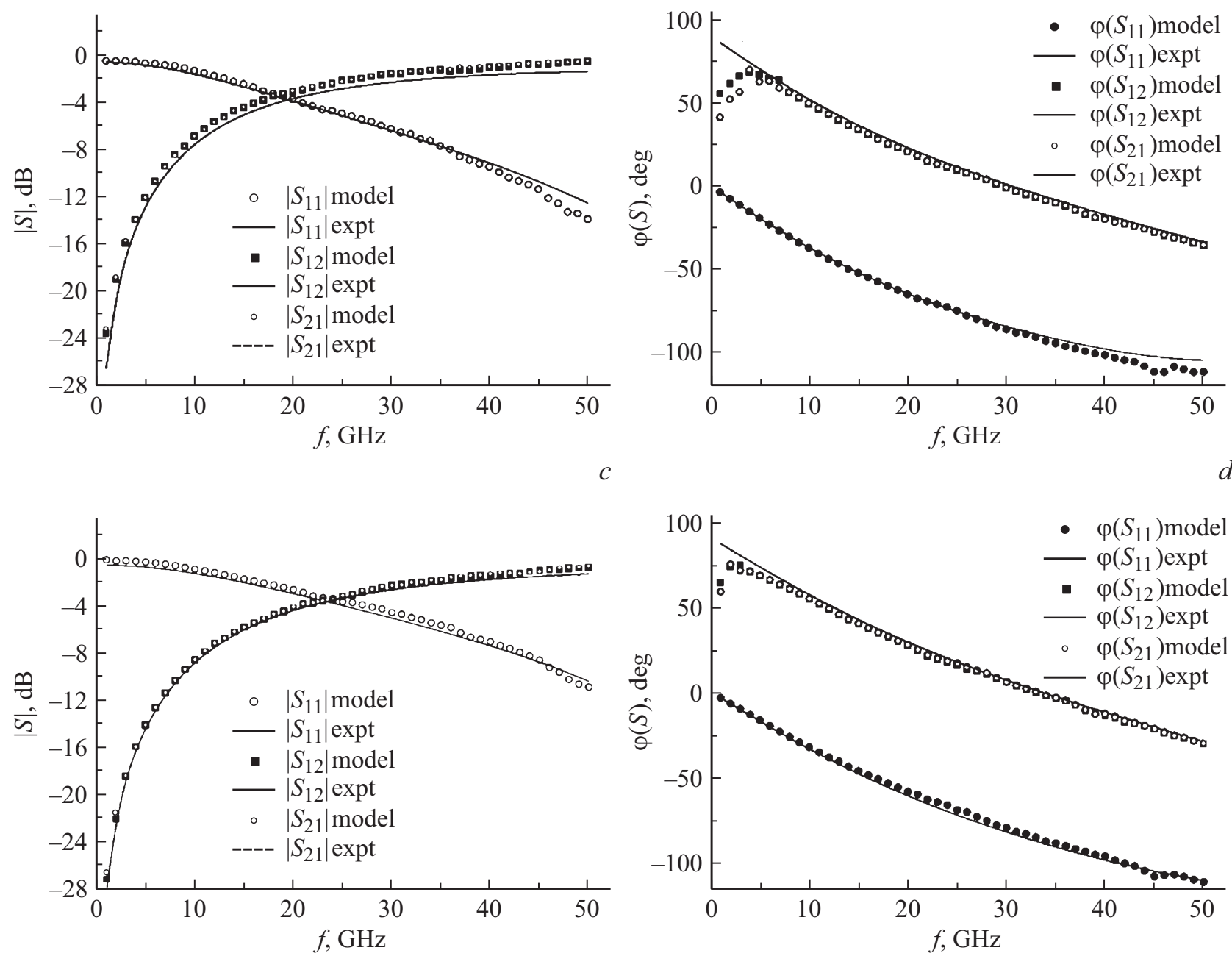

Рис. 7. Частотные зависимости измеренных (сплошные кривые) и смоделированных (точки) амплитудных $|S|$ и фазовых $\varphi(S)$ характеристик при смещениях $U_{f}$ на аноде: $U_{f}= \pm 0.2 \mathrm{~B}(a, b)$ и $U_{r}=-0.6 \mathrm{~B}(c, d)$.

производства Cascade Microtech. Калибровка измерительных трактов портов 1 и 2 осуществлялась по $S$-параметрам согласованием на проход („Thru“), на нагрузку 50 Oм (Load), на холостой ход („Open“) и на короткое замыкание (,Short“). После этого проводилась процедура дэ-эмбеддинга (de-embedding) измеренных $S$-параметров. Суть данной процедуры заключается в исключении влияния на результаты измерения паразитных параметров контактных площадок, линий передач, измерительных зондов и т.д. Для проведения процедуры дэ-эмбеддинга использовалась L-2L методика, подразумевающая использование тестовых элементов в виде 50-омных L (рис. 6, $a$, Line), 2L $\equiv \mathrm{LL}$ (рис. 6, $b$, Line, Line) копланарных линий и $\mathrm{L}-\mathrm{B}_{1} \mathrm{~B}_{2}-\mathrm{L}$ копланарных линий, 
Таблица 1. Значения параметров внутренних элементов диода, полученных с использованием новой дополненной ЭС

\begin{tabular}{c|c|c|c|c|c|c|c}
\hline \multirow{2}{*}{$\begin{array}{c}\text { Внутренние } \\
\text { элементы }\end{array}$} & \multicolumn{7}{|c}{ Смещение на аноде $U, \mathrm{~B}$} \\
\cline { 2 - 8 } & -1 & -0.8 & -0.6 & -0.4 & -0.2 & 0 & +0.2 \\
\hline$C_{j}, \phi \Phi$ & 0.39 & 0.42 & 0.43 & 0.49 & 0.50 & 0.56 & 0.65 \\
$C_{i}, \phi \Phi$ & 0.302 & 0.819 & 1.289 & 5.1879 & 8.095 & 10.478 & 14.526 \\
$R_{b m}$, Ом & 21.781 & 22.499 & 22.446 & 22.448 & 22.349 & 21.697 & 21.774 \\
$R_{l}$, Ом & 11.431 & 11.359 & 10.643 & 10.504 & 10.518 & 10.513 & 11.205 \\
$R_{C}$, Ом & 10.881 & 11.140 & 10.532 & 10.826 & 11.444 & 10.511 & 11.345 \\
$R_{S}$, Ом & 3.53 & 3.60 & 3.49 & 3.50 & 3.54 & 3.44 & 3.54
\end{tabular}

Таблица 2. Значения параметров внутренних элементов диода, полученных с использованием известной ЭС

\begin{tabular}{c|c|c|c|c|c|c|c}
\hline \multirow{2}{*}{$\begin{array}{c}\text { Внутренние } \\
\text { элементы }\end{array}$} & -1 & -0.8 & -0.6 & -0.4 & -0.2 & 0 & +0.2 \\
\cline { 2 - 8 } & 4.9981 & 7.99 & 11.99 & 17.93 & 25.00 & 40.00 & 45.00 \\
$C_{j}$, фФ & 1099.55 & 1087.85 & 1095.98 & 1099.77 & 1092.92 & 1099.96 & 1095.26 \\
$R_{j}$, Ом & 45.60 & 45.62 & 45.09 & 40.04 & 48.00 & 45.01 & 42.01 \\
$R_{S}$, Ом & 4.01 &
\end{tabular}

между которыми в сечение $\mathrm{B}_{1} \mathrm{~B}_{2}$ включен кристалл диода (рис. $6, c)$ с расположенными в сечениях $\mathrm{A}_{1}$ и $\mathrm{A}_{2}$ контактными площадками (рис. 6, Pad) [27,28].

Частотные зависимости амплитудных $|S|$ и фазовых $\varphi(S)$ характеристик малосигнальных параметров рассеяния диодов „Меза-подложка“ для смещений $U_{f}=+0.2$ В и $U_{r}=-0.6$ В после проведения процедуры дэ-эмбеддинга представлены на рис. 7.

\section{5. Экстракция параметров элементов ЭС и верификация результатов}

Восстановление значений (экстракция) параметров элементов ЭС малосигнальных моделей диодных кристаллов по измеренным $S$-параметрам в данной работе осуществлялось с применением комбинированной методики, объединяющей преимущества аналитических и оптимизационных методов экстракции блок-схема алгоритма, которая представлена на рис. 8.

Согласно представленной блок-схеме, процедура экстракции параметров элементов малосигнальной ЭС включает три основных этапа.

На первом этапе проводятся измерения статических и СВЧ-характеристик диодов в „холодных“ (при нулевом напряжении на аноде) и „горячих“ (при положительных и отрицательных смещениях на аноде) режимах. Затем по методу, представленному в [18], с использованием параметров статических BAX определяются внешние паразитные параметры.

На втором этапе определяются $Y$-параметры внутреннего диода путем вычитания внешних элементов по методу, описанному в [18], а также значения внутренних

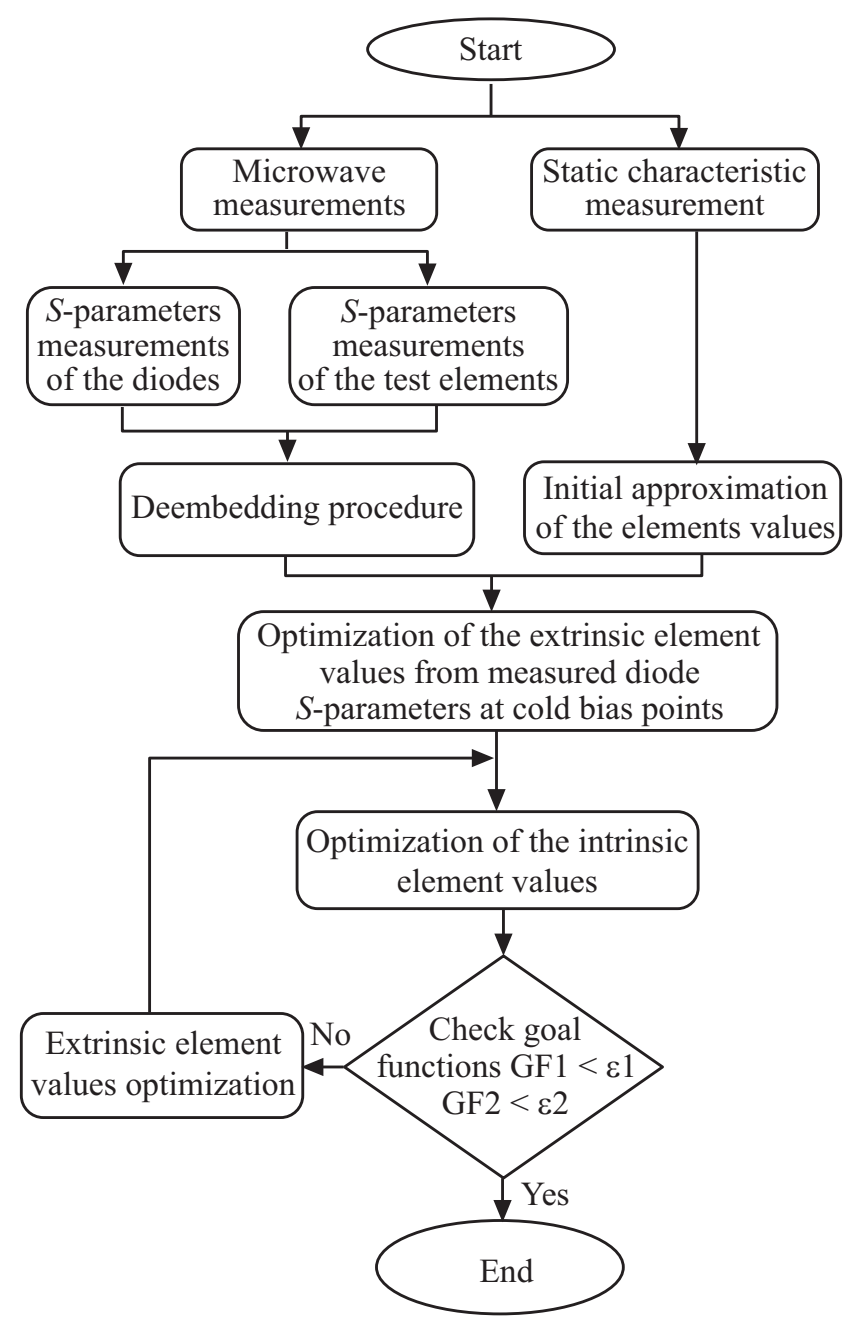

Рис. 8. Блок-схема алгоритма восстановления значений параметров элементов ЭС компактной модели диодного кристалла по малосигнальным $S$-параметрам. 

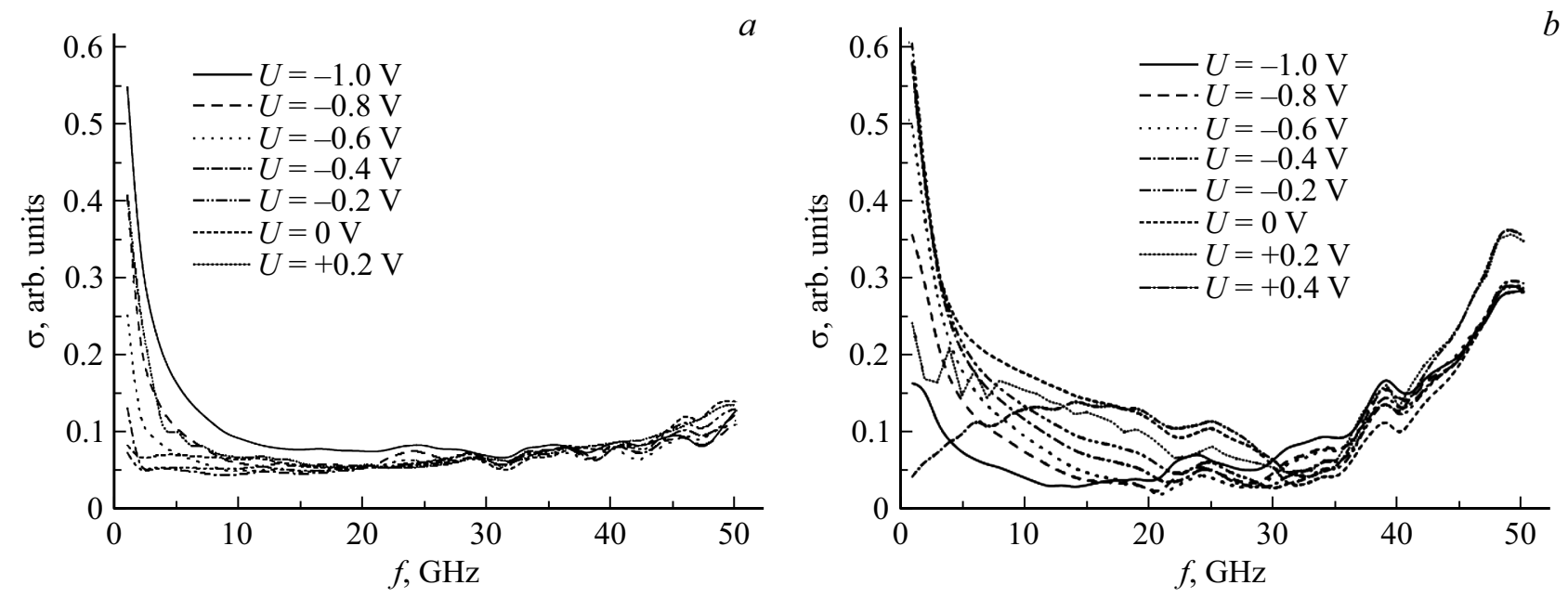

Рис. 9. Частотная зависимость среднеквадратичной погрешности процедуры экстракции значений элементов ЭС при использовании компактных моделей: $a-$ новой и $b-$ известной.

элементов диода. В качестве начального приближения используются значения, найденные из статических ВАХ.

На третьем этапе проводится оптимизация значений элементов эквивалентной схемы - целевых функций GF1 и GF2 для наилучшего соответствия измеренных и смоделированных $S$-параметров. Целевая функция GF1 определяет расхождение между измеренными и смоделированными параметрами, а GF2 - независимость внутренних элементов от частоты.

Результаты верификации показали достаточно хорошее совпадение модельных значений амплитудных $|S|$ и фазовых $\varphi(S)$ СВЧ характеристик с измеренными в контролируемом частотном диапазоне (рис. 7). Использование более совершенной уточненной ЭС позволяет добиться практического совпадения экстрагированных значений статических параметров эквивалентных элементов $C_{j}$ и $R_{S}$ (табл. $\left.1, U=0\right)$ с контролируемыми экспериментально (рис. 5). Процедура оптимизации в этом случае позволяет добиться точности восстановления значений статических параметров ЭС более 95\% (рис. 9, $a$ ).

Значения статических параметров внутренних элементов ЭС исследуемого диода, восстановленные по описанной выше методике (при $U=0 \mathrm{~B}$, табл. 2) с использованием представленной в [18] известной упрощенной компактной модели, значительно отличаются от экспериментальных значений как по емкости $C_{j}=40 \phi \Phi$, так и по последовательному сопротивлению $R_{S}=45.01$ Ом (табл. 2). В этом случае в исследуемом частотном диапазоне процедура оптимизации не позволяет уменьшить погрешность восстановления значений параметров ЭС менее $15 \%$ (рис. 9, $b$ ).

С учетом используемого количества эквивалентных элементов $N$ полученная эмпирическая зависимость $f_{\mathrm{T}}=f_{\mathrm{T}}(N=22) \quad(1)$ позволяет спрогнозировать обусловленный приборным ограничением частотный предел использования предложенной ЭС как $f_{\mathrm{T}}>150$ ТГц. Такое значение $f_{\mathrm{T}}$ уже соизмеримо с физическими ограничениями на предельные частоты функционирования контактов Шоттки $f_{\mathrm{L}}[1,2]$, что указывает на возможность эффективного использования предложенной ЭС в ТГц диапазоне.

Для верификации результатов моделирования в терагерцовом диапазоне необходимо проведение дальнейших исследований с привлечением более высокочастотной техники.

\section{6. Заключение}

Использование электрических выводов в виде воздушных мостов с вискером обеспечивает значительно бо́льшие конструктивно-технологические возможности варьирования форм и размеров кристаллов планарных диодов Шоттки, выполненных по технологиям „Меза-подложка“ и „Меза-Меза“, по сравнению с известными конструкциями балочных диодов с расширенными контактами.

Преимущества предлагаемых конструкций обеспечиваются единым технологическим циклом изготовления металлического усеченного конуса-вискера, соединяющим контакт Шоттки с анодным воздушным выводом диодного кристалла. С одной стороны, это позволяет поднять шляпку анодного контакта и воздушный мост над поверхностью планарной структуры на высоту $h_{W}>d(\mathrm{Au})$ (высота вискера $h_{W}$ превышает толщину $d(\mathrm{Au})$ гальванического утолщения омического контакта) и тем самым значительно снизить паразитные емкости $C_{b r}, C_{b o c}$ и $C_{i}$. С другой стороны, выполнение условия $h_{W}>d(\mathrm{Au})$ позволяет уменьшить расстояние $\Delta x_{R}$ и приблизить контакт Шоттки к омическому контакту, за счет чего достигается значительное уменьшение последовательного сопротивления $R_{S}$. 
Предложенные конструктивные решения одновременно с усовершенствованной малосигнальной эквивалентной схемой позволяют значительно уменьшить влияние приборных ограничений и приблизиться к физическому частотному пределу работы контакта Шоттки $f_{\mathrm{L}}$, повысить надежность диодных кристаллов в СВЧ и КВЧ диапазонах и сократить значительное отставание полупроводниковой электроники от оптоэлектроники в ТГц частотном диапазоне.

\section{Финансирование работы}

Работа выполнялась при финансовой поддержке Министерства образования и науки РФ. Уникальный идентификатор 8.4029.2017/4.6.

\section{Конфликт интересов}

Авторы заявляют об отсутствии конфлика интересов.

\section{Список литературы}

[1] H. Kanaya, F. Hasegawa, E. Yamaka, T. Moriyama, M. Nakajima. JJAP, 28 (4), 544 (1989).

[2] V.G. Ivanov, G.V. Ivanov. Adv. Optoelectron., 2011, 459130 (2011).

[3] P.H. Siegel. IEEE Trans. MTT, 50 (3), 910 (2002).

[4] I. Mehdi, J.V. Siles, C. Lee, E. Schlecht. Proc. IEEE, 105 (6), 990 (2017).

[5] A.Y. Tang. Thesis for The Degree of Doctor of Philosophy (Göteborg, Sweden, Chalmers, 2013).

[6] M.S. Vitiello, G. Scalari, B. Williams, P.D. Natale. Opt. Express, 23 (4), 5168 (2015).

[7] Н.А. Торхов, В.Г. Божков, С.М. Гущин, В.А. Новиков. Тез. докл. 22-й Межсунар. конф. КрыМиКо-2012 (Севастополь, Украина, 2012) с. 635.

[8] Н.А. Торхов, Л.И. Бабак, А.А. Коколов. Тез. докл. 26-й Межсуунар. конф. КрыМиКо-2016 (Севастополь, Россия, 2016) c. 275.

[9] N.A. Torkhov, L.I. Babak, A.A. Kokolov. Dynamics of Systems, Mechanisms and Machines, 1 (2018).

[10] Н.A. Торхов. ФТП, 52 (10), 1150 (2018).

[11] Н.А. Торхов. ФТП, 35 (7), 823(2001).

[12] Интегрированные источники и приемники компании „Virginia Diodes, Inc.“. Электронный ресурс. Режим доступа: http://www.vadiodes.com (дата обращения 04.06.2019).

[13] A. Jelenski, A. Grub, V. Krozer, H.L. Hartnagel. IEEE Trans. Microwave Theory and Techniques, 41 (4), 549 (1993).

[14] J.M. Dieudonn, B. Adelseck, K.E. Schmegner, R. Rittmeyer, A. Colquhoun. IEEE Trans. Microwave Theory and Techniques, 40 (7), 1466 (1992).

[15] Н.А. Торхов. Патент № 2635853 от 16 ноября 2017 г. Приоритет от 26 января 2016 г.

[16] В.С. Арыков, А.Н. Гусев, О.А. Дедкова, А.Ю. Ющенко. Тез. докл. 20-й Межсунар. конф. КрыМиКо-2010 (Севастополь, Украина, 2010) c. 147.

[17] Z. Hu, V.T. Vo, A.A. Rezazadeh. IEEE Micriwave and Wireless Components Lett., 15 (3), 150 (2005).

[18] A.Y. Tang, V. Drakinskiy, K. Yhland et. al. IEEE Trans. Microwave Theory and Techniques, 61 (5), 1870 (2013).
[19] Н.А. Торхов. Патент № 176768 от 29 января 2018 г. Приоритет от 11 февраля 2016 г.

[20] Н.Л. Чуприков. Автореф. докт. дис. (Томск, Россия, ТПГУ, 2010).

[21] E.P. Wigner. Phys. Rev., 98, 145 (1955).

[22] T.E. Hartman. J. Appl. Phys., 33, 3427 (1962).

[23] W. Javorski, D.M. Wardlaw. Phys. Rev. A, 38, 5404 (1988).

[24] C. Bulutay, B.K. Ridley, N.A. Zakhleniuk. Phys. Rev. B, 68, 115205 (2003).

[25] D. Sokolovski, A.Z. Msezane, V.R. Shaginyan. Phys. Rev. A, 71, 064103 (2005).

[26] A.B. Shvartsburg, G. Petite. Optics. Lett., 31, 1127, 2006.

[27] H. Yen, T. Yeh, S. Liu. Progress in Electromagnetics Research Symposium (Beijing, China, 2009) p. 1339.

[28] M. Potereau1, C. Raya, M.D. Matos, S. Fregonese, A. Curutchet, M. Zhang, B. Ardouin, T. Zimmer. J. Comp. Commun., 1, 25 (2013).

Редактор Г.А. Оганесян

\section{The Schottky contact application in the microwave, mm-wave and $\mathrm{THz}$ bands}

\author{
N.A. Torkhov ${ }^{1,2}$, L.I. Babak², A.A. Kokolov ${ }^{2}$ \\ ${ }^{1}$ Scientific-Research Institute of Semiconductors, \\ 634034 Tomsk, Russia \\ ${ }^{2}$ Tomsk State University of Control System \\ and Radioelecrtronics, \\ 634050 Tomsk, Russia
}

\begin{abstract}
The wider range of possibilities to optimize design and electrophysical parameters of Schottky diode dies fabricated in accordance with „Mesa-Substrate“ and „Mesa-Mesa“ planar technologies with anode leads in the form of air bridges with whisker, together with application of more sophisticated compact models give an opportunity to get the best out of Schottky contact physical capacities in designing MICs using diode technologies, to improve their reliability, and to narrow a big gap between semiconductor electronics and optoelectronics in terahertz frequency range.
\end{abstract}

